\title{
More on the locally convex space $(M(X), \beta(X))$ of a locally compact Hausdorff space $X$
}

\author{
Hossein Javanshiri Rasoul Nasr-Isfahani
}

\begin{abstract}
In the previous paper [12] we introduced the definition of the strict topology $\beta(X)$ on the measure space $M(X)$ for a locally compact Hausdorff space $X$. In this paper, we consider on $M(X)$ the topology $\beta(X)$ and we show that $\beta(X)$ is the weak topology under all left multipliers induced by a function space on $M(X)$. We then show that $\beta(X)$ can be considered as a mixed topology. This result is not only of interest in its own right, but also it paves the way to prove that $(M(X), \beta(X))$ is a Mazur space and the locally convex space $(M(S), \beta(S))$, equipped with the convolution multiplication is a complete semitopological algebra, for a wide class of locally compact semigroups $S$.
\end{abstract}

\section{Introduction and preliminaries}

About sixty years passed since two mathematicians introduced two new topologies with different methods. Over the years a considerable amount of work has been done on them and on similar topologies by functional analysts. One of them, Buck [7], investigated the space of continuous functions with the strict topology and the other one, the Polish mathematician Alexiewicz [4], considered a vector space $E$ on which two norms are given and defined a notion of convergence of sequences in $E$, which, in some sense, mixed the topologies given by the two norms. These methods have been studied and generalized by several mathematicians as, for example, Aguayo and his coauthors in [1, 2, 3], Collins [10], Kua [18],

Received by the editors in July 2015.

Communicated by E. Colebunders.

2010 Mathematics Subject Classification : 28C05, 28C15, 46A03, 46H05.

Key words and phrases : Locally compact Hausdorff space, strict topology, locally convex topology, Mazur space, compactly cancellative semigroup, semitopological algebra. 
Choi and Kim [8], Cobzas [9], Katsaras [13, 14, 15, 16], Malkowsky and Velickovic [22], Taylor and his coauthor [23, 24], Wiweger [25, 26] and [12, 19, 20, 21].

Now, let $M(X)$ be the Banach space of all complex Radon measures on the locally compact Hausdorff space $X$ with the total variation norm. Let also, $\mathcal{K}(X)$ denote the set of all compact subsets in $X$. The authors have recently introduced in [12] the strict topology $\beta(X)$ on $M(X)$ and, among other things, they also investigated some attributes of the locally convex topology $\beta(X)$ on $M(X)$. In fact, for any increasing sequence $\left(K_{n}\right)$ in $\mathcal{K}(X)$ and any increasing sequence $\left(\alpha_{n}\right)$ in $\mathbb{R}^{+}$such that $\alpha_{n} \longrightarrow \infty$, they introduced the set of the form

$$
U\left(\left(K_{n}\right),\left(\alpha_{n}\right)\right)=\left\{\mu \in M(X):|\mu|\left(K_{n}\right) \leq \alpha_{n} \text { for all } n \geq 1\right\},
$$

and then they showed that the family $\mathcal{U}(X)$ of all sets of the form $\mathcal{U}\left(\left(K_{n}\right),\left(\alpha_{n}\right)\right)$ is a base of neighbourhoods of zero for a locally convex topology $\beta(X)$ on $M(X)$. In other word, they showed that $\beta(X)$ is the topology generated by the family $\left\{q_{U}: U \in \mathcal{U}(X)\right\}$ of seminorms on $M(X)$, where

$$
q_{U}(\mu)=\sup \left\{\alpha_{n}^{-1}|\mu|\left(K_{n}\right): n \geq 1\right\}
$$

for all $\mu \in M(X)$ and $U:=U\left(\left(K_{n}\right),\left(\alpha_{n}\right)\right) \in \mathcal{U}(X)$.

In this paper, we shall show that the strict topology $\beta(X)$ on $M(X)$ can be viewed as a mixed topology. We then intend to use the theory of mixed topologies to give some general properties of the locally convex space $(M(X), \beta(X))$. This allows us to prove that the locally convex space $(M(X), \beta(X))$ is a Mazur space. Among other thing, we show that $M(X)$ is $\beta(X)$-complete for all locally compact Hausdorff space $X$ and as an application we show that for a wide class of locally compact semigroups $S$, the locally convex space $(M(S), \beta(S))$ with the convolution as a multiplication is a complete semitopological algebra.

\section{The basic results}

We commence this work with the following proposition which shows that we can consider the topology $\beta(X)$ as a weak topology under all left multipliers induced by a function space on $M(X)$. To this end, first we adopt some notations. We denote by $B M(X)$ the Banach space (with the usual norm $\|\cdot\|_{\infty}$ ) of all bounded Borel measurable functions $\varphi$ on $X$. Let also $B M_{0}(X)$ denote the subspace of all functions in $B M(X)$ that vanish at infinity; That is, for $\varepsilon>0$ there exists a compact subset $K$ of $X$ such that $|\varphi(x)|<\varepsilon$ for all $x \in X \backslash K$. Then $M(X)$ is a Banach left $B M_{0}(X)$-module with the module action defined by

$$
\varphi \cdot \mu(B)=\int_{B} \varphi d \mu,
$$

for all $\mu \in M(X), \varphi \in B M_{0}(X)$ and all Borel subset $B$ of $X$. Hence, we can equip $M(X)$ with the strict topology $\tau_{c}$ induced by $B M_{0}(X)$ in the sense of Sentilles and Taylor, that is, the topology generated by the collection of seminorms $\mu \mapsto\|\varphi \cdot \mu\|$ for $\varphi \in B M_{0}(X)$, see [23] for more details. 
Proposition 2.1. Let $X$ be a locally compact Hausdorff space. Then the family $\mathcal{U}(X)$ is a neighborhood base at zero for the topology $\tau_{c}$.

Proof. Let

$$
V=\bigcap_{i=1}^{m}\left\{\mu \in M(X):\left\|\varphi_{i} \cdot \mu\right\|<\varepsilon_{i}\right\},
$$

be a $\tau_{c}$-neighborhood at zero, where $\varphi_{i} \in B M_{0}(X)$ and $\varepsilon_{i}>0$ for $i=1, \cdots, m$. For each $1 \leq i \leq m$, let $\left(C_{i, n}\right)_{n}$ be a sequence of compact subsets of $X$ such that $\left|\varphi_{i}(x)\right|<\varepsilon_{i} / n 2^{n}$ for all $x \in X \backslash C_{i, n}$ and all $n \in \mathbb{N}$. If now, we set

$$
K_{0}=\varnothing \quad \text { and } \quad K_{n}=\bigcup_{i=1}^{m} C_{i, n} \quad(n \in \mathbb{N}),
$$

then for all $x \in X \backslash K_{n}$ and all $1 \leq i \leq m$, we can see that $\left|\varphi_{i}(x)\right|<\varepsilon_{i} / n 2^{n}$. Moreover, if

$$
\gamma_{1}:=\frac{\min \left\{\varepsilon_{1}, \cdots, \varepsilon_{m}\right\}}{3\left(\left\|\varphi_{1}\right\|_{\infty}+\cdots+\left\|\varphi_{m}\right\|_{\infty}+1\right)},
$$

and $\gamma_{n}:=(n-1) / 2$ for all $n \geq 2$, then $U\left(\left(K_{n}\right),\left(\gamma_{n}\right)\right) \subseteq V$; Indeed, for a $\mu$ in the set $U\left(\left(K_{n}\right),\left(\gamma_{n}\right)\right)$ and $i=1, \cdots, m$, we have

$$
\begin{aligned}
\left\|\varphi_{i} \cdot \mu\right\|=\left|\varphi_{i} \cdot \mu\right|\left(\bigcup_{n=1}^{\infty} K_{n}\right) & =\lim _{n \rightarrow \infty}\left|\varphi_{i} \cdot \mu\right|\left(K_{n}\right) \\
& =\lim _{n \rightarrow \infty} \sum_{j=1}^{n}\left|\varphi_{i} \cdot \mu\right|\left(K_{j} \backslash K_{j-1}\right) \\
& \leq \int_{K_{1}}\left|\varphi_{i}\right| d|\mu|+\lim _{n \rightarrow \infty} \sum_{j=2}^{n} \int_{K_{j} \backslash K_{j-1}}\left|\varphi_{i}\right| d|\mu| \\
& \leq\left\|\varphi_{i}\right\|_{\infty} \gamma_{1}+\lim _{n \rightarrow \infty} \sum_{j=2}^{n} \frac{\varepsilon_{i}}{(j-1) 2^{(j-1)}}|\mu|\left(K_{j} \backslash K_{j-1}\right) \\
& \leq\left\|\varphi_{i}\right\|_{\infty} \gamma_{1}+\lim _{n \rightarrow \infty} \sum_{j=2}^{n} \frac{\gamma_{j} \varepsilon_{i}}{(j-1) 2^{(j-1)}} \\
& \leq\left\|\varphi_{i}\right\|_{\infty} \gamma_{1}+\lim _{n \rightarrow \infty} \sum_{j=2}^{n} \frac{\varepsilon_{i}}{2^{j}}<\varepsilon_{i} .
\end{aligned}
$$

Conversely, let $\mathcal{U}\left(\left(K_{n}\right),\left(\alpha_{n}\right)\right)$ be an arbitrary element of $\mathcal{U}(X)$. Consider the function

$$
\varphi:=\sum_{n=1}^{\infty} \frac{1}{\alpha_{n}} \chi_{K_{n} \backslash K_{n-1}}
$$

in $B M_{0}(X)$, where $\chi_{K_{n} \backslash K_{n-1}}$ denotes the characteristic function of $K_{n} \backslash K_{n-1}$ on $X$ and $K_{0}=\varnothing$. Then

$$
V:=\{\mu \in M(X):\|\varphi \cdot \mu\| \leq 1\},
$$


is a $\tau_{c}$-neighborhood of zero such that $V \subseteq U\left(\left(K_{n}\right),\left(\alpha_{n}\right)\right)$; Indeed, for each $\mu \in V$ we have

$$
\begin{aligned}
|\mu|\left(K_{n}\right) & =|\mu|\left(\bigcup_{j=1}^{n}\left(K_{j} \backslash K_{j-1}\right)\right) \\
& \leq \sum_{j=1}^{n} \frac{\alpha_{n}}{\alpha_{j}}|\mu|\left(K_{j} \backslash K_{j-1}\right) \\
& \leq \alpha_{n} \int_{X} \varphi d|\mu| \leq \alpha_{n}
\end{aligned}
$$

for all $n \in \mathbb{N}$, and this completes the proof.

Apart from the locally convex topology $\beta(X)$ on $M(X)$, there are two other locally convex structures on the space $M(X)$ whose definitions are as follows. By $n(X)$ we denote the topology generated by the total variation norm on $M(X)$, and by $\kappa(X)$ we denote the second locally convex structure on $M(X)$ which is generated by the seminorms

$$
\mathcal{P}_{K}(\mu)=|\mu|(K),
$$

where $K$ runs over all compact subsets of $X$. Our main result in this section is to show that the strict topology on $M(X)$ can be constructed from $\kappa(X)$ and $n(X)$. To this end, we state first some of the standard definitions which will be used in the sequel and we define the mixed topology.

A $D F$-space is a locally convex space $E$ which possesses a fundamental sequence $\left(B_{n}\right)_{n}$ of bounded sets and has the property that if $\left(U_{n}\right)_{n}$ is a sequence of closed, absolutely convex neighborhoods of zero so that $U=\bigcap_{n=1}^{\infty} U_{n}$ absorbs bounded sets of $E$, then $U$ is also a neighborhood of zero. Let $E$ be a vector space with two locally convex topologies $\tau$ and $\tau^{*}$ satisfying:

(i) $\tau^{*} \leq \tau$;

(ii) $(E, \tau)$ is a $D F$-space with a base $\left(B_{n}\right)$ of absolutely convex bounded sets such that

$$
B_{n}+B_{n} \subseteq B_{n+1} \text { for each } n \text {; }
$$

(iii) each $B_{n}$ is $\tau^{*}$-closed.

For any sequence $\left(U_{n}^{*}\right)$ of absolutely convex neighborhoods of zero in $\left(E, \tau^{*}\right)$ set

$$
U^{\gamma}=\bigcup_{n=1}^{\infty}\left(U_{1}^{*} \cap B_{1}+\cdots+U_{n}^{*} \cap B_{n}\right) .
$$

It is easy to see that the set of all $U^{\gamma}$ forms a base of neighborhoods of zero for a locally convex topology $\gamma:=\gamma\left[\tau, \tau^{*}\right]$ on $E$. As usual, we call this topology the mixed topology on $E$. We refer the reader to the references $[4,25,26]$ for more information about the theory of mixed topology.

By a method similar to that of [19, Proposition 2.1] one can easily obtain the following generalization of that theorem. The details are omitted.

Proposition 2.2. Let $X$ be a locally compact Hausdorff space. Then the topology $\beta(X)$ on $M(X)$ is the mixed topology $\gamma(X)=\gamma[\kappa(X), n(X)]$. 
An argument similar to the proof of [19, Theorem 2.2] with the aid of Proposition 2.2 gives the following generalization of that Theorem.

Theorem 2.3. Let $X$ be a locally compact Hausdorff space. Then the following assertions hold.

(i) A subset of $M(X)$ is $n(X)$-bounded if and only if it is $\beta(X)$-bounded.

(ii) On $n(X)$-bounded subsets of $M(X), \beta(X)=\kappa(X)$.

(iii) A sequence in $M(X)$ is $\beta(X)$-convergent to zero if and only if it is $n(X)$-bounded and $\kappa(X)$-convergent to zero.

(iv) A linear map from $(M(X), \beta(X))$ into a locally convex space is continuous if and only if its restriction to $n(X)$-bounded sets is continuous for $\kappa(X)$.

$(\mathrm{v}) \beta(X)$ is the finest locally convex topology on $M(X)$ which agrees with $\kappa(X)$ on $n(X)$-bounded sets of $M(X)$.

(vi) A subset of $M(X)$ is $\beta(X)$-compact if and only if it is $n(X)$-bounded and $\kappa(X)$-compact.

(vii) $(M(X), \beta(X))$ is complete if and only if each $n(X)$-bounded set is $\kappa(X)$-complete.

Recall that a locally convex space $(E, \tau)$ is a Mazur space if every sequentially $\tau$-continuous linear functional on $E$ is $\tau$-continuous. In the next result, we deal with this property for the locally convex space $(M(X), \beta(X))$. First, we recall some notations from [11, 12]. For $\mu \in M(X)$, let $L^{\infty}(|\mu|)$ denote the Banach space of all bounded Borel $\mu$-measurable functions $F(\mu)$ on $X$ with the essential supremum norm

$$
\|F(\mu)\|_{\mu, \infty}:=\inf \{\alpha \geq 0:\{x \in X:|F(\mu)(x)|>\alpha\} \text { is }|\mu|-\text { null }\} .
$$

It follows from this definition that the inequality $|F(\mu)(x)|<\varepsilon$ holds for $\mu$-almost all $x$ if and only if $\|F(\mu)\|_{\mu, \infty}<\varepsilon$. Define $L^{\infty}(M(X))$ to be the set of all elements $F$ in $\Pi\left\{L^{\infty}(|\mu|): \mu \in M(X)\right\}$ such that $F(\mu)=F(v)$ a.e. $[\mu]$ for all $\mu, v \in M(X)$ with $\mu \ll v$. Then $\sup _{\mu \in M(X)}\|F(\mu)\|_{\mu, \infty}<\infty$ for all $F \in L^{\infty}(M(X))$ and $L^{\infty}(M(X))$ is a Banach space with norm $\|F\|=\sup _{\mu \in M(X)}\|F(\mu)\|_{\mu, \infty}$, see [11, Lemma 5.11, page 76]. If now, for arbitrary $F \in L^{\infty}(M(X))$, we define the functional $\Phi_{F}: M(X) \rightarrow \mathbb{C}$ by

$$
\Phi_{F}(\mu)=\int_{X} F(\mu) d \mu,
$$

for all $\mu \in M(X)$, then by an elegant use of the Radon-Nikodym Theorem one can see that the map $F \mapsto \Phi_{F}$ is an isometric isomorphism from $L^{\infty}(M(X))$ onto $(M(X), n(X))^{*}$, see [11, Theorem 5.12]. Moreover, we recall from [12] that a functional $F \in L^{\infty}(M(X))$ vanishes at infinity if for each $\varepsilon>0$, there is a compact subset $K_{\varepsilon}$ of $X$ such that for each $\mu \in M(X),|F(\mu)(x)|<\varepsilon$ for $|\mu|$-almost all $x \in X \backslash K_{\varepsilon}$; Formally

$$
\begin{aligned}
\forall \varepsilon>0 \quad \exists K_{\varepsilon} \in \mathcal{K}(X) \quad \text { s.t. } \quad \forall \mu \in M(X), \quad & |F(\mu)(x)|<\varepsilon \\
& \text { for }|\mu|-\text { almost all } x \in X \backslash K_{\varepsilon} .
\end{aligned}
$$

We denote by $L_{0}^{\infty}(M(X))$ the subspace of $L^{\infty}(M(X))$ consisting of all $F \in L^{\infty}(M(X))$ that vanish at infinity. In our previous work [12], among other things, we showed that the strong dual of $(M(X), \beta(X))$ can be identified with $L_{0}^{\infty}(M(X))$. 
Proposition 2.4. Let $X$ be a locally compact Hausdorff space. Then the locally convex space $(M(X), \beta(X))$ is a Mazur space.

Proof. Let $L$ be a sequentially $\beta(X)$-continuous linear functional on $M(X)$. Therefore, there exists an $F \in L^{\infty}(M(X))=(M(X), n(X))^{*}$ such that $\Phi_{F}=L$. Hence, according to [12, Theorem 3.2], it suffices to show that $F$ is in $L_{0}^{\infty}(M(X))$. We divide the proof in three cases as follows.

Case 1. $X$ is compact. In this case, the Proposition 4.1 of [12] implies that $\beta(X)=n(X)$. Moreover, $L_{0}^{\infty}(M(X))=L^{\infty}(M(X))$. Hence $F \in L_{0}^{\infty}(M(X))$.

Case 2. $X$ is $\sigma$-compact Hausdorff space which is not compact. In this case, there exists an increasing sequence $\left(K_{n}\right) \subseteq \mathcal{K}(X)$ with $X=\bigcup_{n=1}^{\infty} K_{n}$ satisfying the condition that each $K$ in $\mathcal{K}(X)$ is contained in some $K_{n}$. Also by Urysohn's Lemma and Riesz Representation Theorem ([11, Theorem 5.7, page 75]), for each $n \geq 1$ we can find $\mu_{n} \in M(X)$ such that $\left|\mu_{n}\right|\left(K_{n}\right)>0$. In particular $\left|\mu_{n}\right|\left(X \backslash K_{n}\right)>0$ for each $n \in \mathbb{N}$. So, if we set

$$
v=\sum_{n=1}^{\infty} 2^{-n}\left|\mu_{n}\right| /\left\|\mu_{n}\right\|,
$$

then $v\left(K_{n}\right)$ and $v\left(X \backslash K_{n}\right)$ are nonzero for all $n \geq 1$. Now, suppose on the contrary that $F \notin L_{0}^{\infty}(M(X))$. Then, there is a number $\varepsilon_{0}>0$ such that $\left\|F(\mu) \chi_{X \backslash K}\right\|_{\mu, \infty}>$ $\varepsilon_{0}$ for all $K \in \mathcal{K}(X)$ and all $\mu \in M(X)$. In particular, $\left\|F(v) \chi_{X \backslash K_{n}}\right\|_{\nu, \infty}>\varepsilon_{0}$ for all $n \in \mathbb{N}$. It follows that there exists a sequence $F_{n}^{\prime}(v)$ in $L^{1}(v)$ which is bounded by one and

$$
\left|\int_{X} \chi_{X \backslash K_{n}} F_{n}^{\prime}(v) F(v) d v\right|>\varepsilon_{0} .
$$

If now, for each $n \in \mathbb{N}$, we set $v_{n}:=\chi_{X \backslash K_{n}} F_{n}^{\prime}(v) d v$, then $v_{n}$ is in $M(X)$ and $v_{n} \ll v$. Moreover, for arbitrary compact subset $K$ of $X$, we see that $\left|v_{n}\right|(K)$ tends to zero. Hence, by Theorem 2.2(iii), the sequence $\left(v_{n}\right)$ converges to zero with respect to the topology $\beta(X)$. Therefore, the $\beta(X)$-sequential continuity of the functional $L$ implies that $L\left(v_{n}\right)$ tends to zero. But this contradicts (2); This is because of, $F\left(v_{n}\right)=F(v)$ a.e. $\left[v_{n}\right]$ for all $n \in \mathbb{N}$ and therefore

$$
\begin{aligned}
\left|L\left(v_{n}\right)\right| & =\left|\int_{X} F\left(v_{n}\right) d v_{n}\right| \\
& =\left|\int_{X} F(v) d v_{n}\right| \\
& =\left|\int_{X} \chi_{X \backslash K_{n}} F_{n}^{\prime}(v) F(v) d v\right| .
\end{aligned}
$$

Case 3. $X$ is a locally compact Hausdorff space which is not $\sigma$-compact. In this case, there exists a sequence $\left(V_{n}\right)$ of relatively compact open subsets of $X$ such that the sets $V_{n}$ for all $n \geq 1$ are pairwise disjoint and $V_{n} \subseteq X \backslash K_{0}$, where $K_{0}$ is a fixed nonempty compact subset of $X$. It follows that for any compact subset $K$ of $X$ the sets $V_{n}$ eventually do not intersect $K$. Also by Urysohn's Lemma and Riesz Representation Theorem ([11, Theorem 5.7, page 75]), for each $n \geq 1$ we can find $\mu_{n} \in M(X)$ such that $\left|\mu_{n}\right|\left(V_{n}\right)>0$. So, if we set

$$
v=\sum_{n=1}^{\infty} 2^{-n}\left|\mu_{n}\right| /\left\|\mu_{n}\right\|,
$$


then $v\left(V_{n}\right) \neq 0$ for all $n \geq 1$. Now, suppose on the contrary that $F \notin L_{0}^{\infty}(M(X))$. Then, there is a number $\varepsilon_{0}>0$ such that $\left\|F(\mu) \chi_{X \backslash K}\right\|_{\mu, \infty}>\varepsilon_{0}$ for all $K \in \mathcal{K}(X)$ and all $\mu \in M(X)$. In particular, $\left\|F(v) \chi_{X \backslash K_{0}}\right\|_{\nu, \infty}>\varepsilon_{0}$. Therefore, $\left\|F(v) \chi_{V_{n}}\right\|_{v, \infty}>$ $\varepsilon_{0}$ for all $n \in \mathbb{N}$. It follows that there exists a sequence $F_{n}^{\prime}(v)$ in $L^{1}(v)$ which is bounded by one and

$$
\left|\int_{X} \chi_{V_{n}} F_{n}^{\prime}(v) F(v) d v\right|>\varepsilon_{0} .
$$

If now, for each $n \in \mathbb{N}$, we set $v_{n}:=\chi_{V_{n}} F_{n}^{\prime}(v) d v$, then the proof of this case will be completed by the same argument as in the proof of the Case 2 .

We now show that for a locally compact Hausdorff space $X$, the locally convex space $(M(X), \beta(X))$ is complete. For this, let us recall that from [6, Definition 1.2, page 17], a set function $\lambda: \mathcal{K}(X) \rightarrow[0, \infty)$ is called a Radon content if

$$
\lambda\left(K_{2}\right)-\lambda\left(K_{1}\right)=\sup \left\{\lambda(K): K \subseteq K_{2} \backslash K_{1}, K \in \mathcal{K}(X)\right\}
$$

for all $K_{1}, K_{2} \in \mathcal{K}(X)$ with $K_{1} \subseteq K_{2}$.

Proposition 2.5. Let $X$ be a locally compact Hausdorff space. Then $(M(X), \beta(X))$ is a complete topological space.

Proof. In view of Theorem 2.3 (vii), it suffices to show that each $n(X)$-bounded set is $\kappa(X)$-complete. So, let $\left(\mu_{\alpha}\right)$ be an $n(X)$-bounded net which is Cauchy in the $\kappa(X)$-topology, then $\left(\chi_{K} \cdot \mu_{\alpha}\right)$ is an $n(X)$-Cauchy net in $M(X)$ for each compact subset $K \subseteq X$. For each $K \in \mathcal{K}(X)$, let $\mu_{K}$ be the limit of the net $\left(\chi_{K} \cdot \mu_{\alpha}\right)$ with respect to the norm topology of $M(X)$. The proof will be complete if we show that there is a $\mu \in M(X)$ such that $\chi_{K} \cdot \mu=\mu_{K}$ for all compact subsets $K$. To this end, without loss of generality, we may assume that $\mu_{K} \geq 0$ for all compact sets $K$. Now, define $\lambda: \mathcal{K}(X) \rightarrow[0, \infty)$ by

$$
\lambda(K):=\mu_{K}(K)
$$

for all $K \in \mathcal{K}(X)$. If $K_{1}$ and $K_{2}$ are two arbitrary compact subsets of $X$ with $K_{1} \subseteq K_{2}$. Then, for each compact subset $K$ of $X$ such that $K \subseteq K_{2}$, we have

$$
\begin{aligned}
\mu_{K_{2}}(K) & =\lim _{\alpha} \chi_{K_{2}} \cdot \mu_{\alpha}(K) \\
& =\lim _{\alpha} \int_{K} \chi_{K_{2}} d \mu_{\alpha} \\
& =\lim _{\alpha} \int_{K} \chi_{K} d \mu_{\alpha} \\
& =\lim _{\alpha} \chi_{K} \cdot \mu_{\alpha}(K)=\mu_{K}(K) .
\end{aligned}
$$

In particular, $\mu_{K_{2}}\left(K_{1}\right)=\mu_{K_{1}}\left(K_{1}\right)$ and therefore,

$$
\lambda\left(K_{2}\right)-\lambda\left(K_{1}\right)=\mu_{K_{2}}\left(K_{2}\right)-\mu_{K_{1}}\left(K_{1}\right)=\mu_{K_{2}}\left(K_{2} \backslash K_{1}\right) .
$$


Hence the regularity of the measure $\mu_{K_{2}} \in M(X)$ together with the fact that $\mu_{K_{2}}(K)=\mu_{K}(K)$ when $K \subseteq K_{2} \backslash K_{1} \subseteq K_{2}$, implies that

$$
\begin{aligned}
\lambda\left(K_{2}\right)-\lambda\left(K_{1}\right) & =\sup \left\{\mu_{K_{2}}(K): K \subseteq K_{2} \backslash K_{1}, K \in \mathcal{K}(X)\right\} \\
& =\sup \left\{\mu_{K}(K): K \subseteq K_{2} \backslash K_{1}, K \in \mathcal{K}(X)\right\} \\
& =\sup \left\{\lambda(K): K \subseteq K_{2} \backslash K_{1}, K \in \mathcal{K}(X)\right\} .
\end{aligned}
$$

Thus $\lambda$ is a Radon content set function on $\mathcal{K}(X)$. Therefore, if we define

$$
\mu(A):=\sup \{\lambda(K): K \subseteq A, K \in \mathcal{K}(X)\}, \quad(A \subseteq X)
$$

then by Theorem 2.1.4 in [6], the restriction of $\mu$ to the $\sigma$-algebra of all Borel subsets is a Radon measure on $X$.

Now, the proof will be completed by showing that $\chi_{K} \cdot \mu=\mu_{K}$ for all $K \in \mathcal{K}(X)$; This is because of, the validity of this equality for each compact subset $K$ of $X$, implies that $\left(\mu_{\alpha}\right)$ tends to $\mu$ with respect to the topology $\kappa(X)$ on $M(X)$. To that end, suppose that $K$ is an arbitrary compact subset of $X$. Then by the same argument as above for each $C \in \mathcal{K}(X)$, we can see that

$$
\mu_{K \cap C}(K \cap C)=\mu_{K}(K \cap C),
$$

and therefore

$$
\begin{aligned}
\chi_{K} \cdot \mu(C) & =\mu(K \cap C) \\
& =\sup \left\{\lambda\left(K^{\prime}\right): K^{\prime} \subseteq K \cap C, K^{\prime} \in \mathcal{K}(X)\right\} \\
& =\sup \left\{\mu_{K^{\prime}}\left(K^{\prime}\right): K^{\prime} \subseteq K \cap C, K^{\prime} \in \mathcal{K}(X)\right\} \\
& =\sup \left\{\mu_{K \cap C}\left(K^{\prime}\right): K^{\prime} \subseteq K \cap C, K^{\prime} \in \mathcal{K}(X)\right\} \\
& =\mu_{K \cap C}(K \cap C) \\
& =\mu_{K}(K \cap C) \\
& =\lim _{\alpha} \chi_{K} \cdot \mu_{\alpha}(K \cap C) \\
& =\lim _{\alpha} \int_{K \cap C} \chi_{K} d \mu_{\alpha} \\
& =\lim _{\alpha} \int_{C} \chi_{K} d \mu_{\alpha} \\
& =\mu_{K}(C)
\end{aligned}
$$

where in the fourth equality we use the fact that

$$
\mu_{K^{\prime}}\left(K^{\prime}\right)=\mu_{K \cap C}\left(K^{\prime}\right)
$$

for all $K^{\prime} \in \mathcal{K}(X)$ with $K^{\prime} \subseteq K \cap C$. Hence $\chi_{K} \cdot \mu(C)=\mu_{K}(C)$ for all $C \in \mathcal{K}(X)$. We now invoke the regularity of the measures $\chi_{K} \cdot \mu$ and $\mu_{K}$, to conclude that $\chi_{K} \cdot \mu=\mu_{K}$. This completes the proof of the proposition. 


\section{An application to semigroups}

Let $S$ denote a locally compact semigroup; That is, a semigroup with a locally compact Hausdorff topology under which the binary operation of $S$ is jointly continuous. The convolution multiplication "*” on $M(S)$ is defined by

$$
\langle\mu * v, f\rangle=\int_{S} \int_{S} f(x y) d \mu(x) d v(y)
$$

for all $f \in C_{0}(S)$, where $C_{0}(S)$ is the Banach space of all bounded complex-valued continuous functions on $S$ vanishing at infinity. In particular, for all Borel sets $B$ of $S$ we have

$$
\begin{aligned}
\mu * v(B) & =\int_{S} \mu\left(B x^{-1}\right) d v(x) \\
& =\int_{S} v\left(y^{-1} B\right) d \mu(y) ;
\end{aligned}
$$

where $y^{-1} B:=\{t \in S: y t \in B\}$ and $B x^{-1}:=\{t \in S: t x \in B\}$; see [5] for more details.

We are now in position to show that the convolution multiplication on $M(S)$ is $\beta(S)$-separately continuous for a wide class of locally compact semigroups which contains locally compact groups and discrete semigroups as elementary examples. To this end, let us recall that a locally compact semigroup $S$ is called compactly cancellative if $C^{-1} D$ and $C D^{-1}$ are compact for all compact subsets $C$ and $D$ of $S$, where

$$
\begin{aligned}
& C^{-1} D=\{s \in S: c s \in D \text { for some } c \in C\} ; \\
& C D^{-1}=\{s \in S: \text { sd } \in C \text { for some } d \in D\} .
\end{aligned}
$$

Theorem 3.1. Let $S$ be a compactly cancellative semigroup with identity. Then the locally convex space $(M(S), \beta(S))$ with the convolution multiplication is a complete semitopological algebra.

Proof. The completeness follows from Proposition 2.5. Now, in view of parts (ii) and (iv) of Theorem 2.3, we only need to show that the convolution multiplication on $M(S)$ is $\beta(S)$-continuous on $n(S)$-bounded subsets. To this end, let $\left(\mu_{l}\right)$ be a norm bounded net in $M(S)$ convergent to zero in $\beta(S)$ and let $v \in M(S)$ with $\|v\|>0$. Suppose that $U\left(\left(K_{n}\right),\left(\alpha_{n}\right)\right)$ is an arbitrary $\beta(S)$-neighborhood of zero and $K \in K(\mathcal{S})$ is chosen so that $|v|(S \backslash K)<\alpha_{1} / 2 M$, where $M$ is the bound of the net $\left(\mu_{l}\right)$. If now, we set

$$
K_{n}^{\prime}:=K_{n} K^{-1} \quad \text { and } \quad \alpha_{n}^{\prime}:=\frac{\alpha_{n}}{2\|v\|},
$$

then there exists $\iota_{0}$ such that $\mu_{\iota} \in U\left(\left(K_{n}^{\prime}\right),\left(\alpha_{n}^{\prime}\right)\right)$ for all $\iota \geq \iota_{0}$. Now, we can write

$$
\begin{aligned}
\left|\mu_{\iota} * v\right|\left(K_{n}\right) & \leq\left|\mu_{\iota}\right| *|v|\left(K_{n}\right) \\
& =\int_{K}\left|\mu_{\iota}\right|\left(K_{n} t^{-1}\right) d|v|(t)+\int_{S \backslash K}\left|\mu_{\iota}\right|\left(K_{n} t^{-1}\right) d|v|(t) \\
& \leq\left|\mu_{\iota}\right|\left(K_{n}^{\prime}\right) \int_{K} d|v|(t)+M \int_{S \backslash K} d|v|(t) \\
& \leq\|v\| \alpha_{n}^{\prime}+M\left(\frac{r_{1}}{2 M}\right) \leq r_{n}
\end{aligned}
$$


for all $\iota \geq \iota_{0}$. Hence, $\mu_{\iota} * v \longrightarrow 0$ in the $\beta(S)$-topology.

Acknowledgments. The authors would like to express his gratitude to Professor Saeid Maghsoudi for reading and commenting on the first draft of this paper.

\section{References}

[1] J. Aguayo, Strict topologies on spaces of continuous functions and $u$-additive measure spaces, J. Math. Anal. Appl. 220 (1998), 77-89.

[2] J. Aguayo, A. K. Katsaras and S. Navarro, On the dual space for the strict topology $\beta_{1}$ and the space $M(X)$ in function space, Ultrametric functional analysis, 15-37, Contemp. Math., 384, Amer. Math. Soc., Providence, RI, 2005.

[3] J. Aguayo, S. Navarro and J. Ojeda, A generalized inductive limit strict topology on the space of bounded continuous functions, Indag. Math. (N.S.) 18 (2007), 485-494.

[4] A. Alexiewicz, On the two-norm convergence, Studia Math. 14 (1953), 49-56.

[5] A. C. Baker and J. W. Baker, Algebra of measures on a locally compact semigroup III, J. Lond. Math. Soc. 4 (1972), 685-695.

[6] C. Berg, J. P. R. Christensen and P. Ressel, Harmonic Analysis on Semigroups. Graduate Texts in Mathematics, 100, Springer, New York (1984).

[7] R. C. Buck, Bounded continuous functions on a locally compact space, Michigan Math. J. 5 (1958), 95-104.

[8] Y. S. Choi and J. M. Kim, The dual space of $\left(L(X, Y), \tau_{p}\right)$ and the p-approximation property, J. Funct. Anal. 259 (2010), 2437-2454.

[9] S. Cobzas, Compact and precompact sets in asymmetric locally convex spaces, Topology Appl. 156 (2009), 1620-1629.

[10] H. S. Collins, On the space $l^{\infty}(S)$ with the strict topology, Math. Z. 106 (1968), 361-373.

[11] J. B. Conway, A course in functional analysis, Springer-Verlag, New York, (1985).

[12] H. Javanshiri and R. Nasr-Isfahani, The strict topology for the space of Radon measures on a locally compact Hausdorff space, Topology Appl. 160 (2013), 887-895.

[13] A. K. Katsaras, On the strict topology in non-Archimedean spaces of continuous functions, Glas. Mat. Ser. III 35 (2000), 283-305. 
[14] A. K. Katsaras, On the strict topology in the locally convex setting, Math. Ann. 216 (1975), 105-111.

[15] A. K. Katsaras, On the strict topology in the nonlocally convex setting II, Acta Math. Hungar. 41 (1983), 77-88.

[16] A. K. Katsaras, Separable measures and strict topologies on spaces of nonArchimedean valued functions, Bull. Belg. Math. Soc. 9 (2002), 117-139.

[17] L. A. Khan, Topological modules of continuous homomorphisms, J. Math. Anal. Appl. 343 (2008), 141-150.

[18] R. T. Kuo, A strict topology on $L^{\infty}(\Omega, \Sigma, \mu, X)$, Soochow J. Math. 9 (1983), $111-125$.

[19] S. Maghsoudi, A mixed topology over the space of measures with continuous translations on a locally compact semigroup, Semigroup Forum 86 (2013), 133-139.

[20] S. Maghsoudi and R. Nasr-Isfahani, Strict topology on the discrete Lebesgue spaces, Bull. Aust. Math. Soc. 83 (2011), 241-255.

[21] S. Maghsoudi and R. Nasr-Isfahani, Strict topology as a mixed topology on Lebesgue spaces, Bull. Aust. Math. Soc. 84 (2011), 504-515.

[22] E. Malkowsky and V. Velickovic, Topologies of some new sequence space, their duals, and the graphical representations of neighborhoods, Topology Appl. 158 (2011), 13691380.

[23] F. D. Sentilles and D. C. Taylor, Factorization in Banach algebras and the general strict topology, Trans. Amer. Math. Soc. 142 (1969), 141-152.

[24] D. C. Taylor, The strict topology for double centralizer algebras, Trans. Amer. Math. Soc. 150 (1970), 633-643.

[25] A. Wiweger, A topologisation of Saks spaces, Bull. Acad. Polon. Sei. 5 (1957), 773-777.

[26] A. Wiweger, Linear spaces with mixed topology, Studia Math. 20 (1961), $47-68$.

Department of Mathematics, Yazd University,

P.O. Box: 89195-741, Yazd, Iran,

e-mail: h.javanshiri@yazd.ac.ir

Department of Mathematical Sciences, Isfahan University of Technology,

Isfahan 84156-83111, Iran,

e-mail: isfahani@cc.iut.ac.ir 